\title{
STABLE EQUIVALENCE FOR SOME CATEGORIES WITH RADICAL SQUARE ZERO
}

\author{
BY
}

\section{IDUN REITEN}

\begin{abstract}
For certain abelian categories with radical square zero, containing artin rings with radical square zero as a special case, we give a way of constructing hereditary abelian categories stably equivalent to them, i.e. such that their categories modulo projectives are equivalent categories.
\end{abstract}

Introduction. Let $\mathbf{C}$ be an additive category where idempotents split. Denote as in [2] by Mod $\mathbf{C}$ the category of contravariant additive functors from $\mathbf{C}$ to abelian groups (called $\mathbf{C}$-modules), and by mod $\mathbf{C}$ the full subcategory of finitely presented functors. We shall further assume that each object in $\mathbf{C}$ is a finite direct sum of indecomposable objects in $\mathrm{C}$, and that the endomorphism ring $R_{C}$ of an indecomposable object $C$ is a division ring modulo its radical, so that a decomposition of an object in $\mathbf{C}$ into a finite direct sum of indecomposable objects is unique. Assume also that $\mathbf{D}=\bmod \mathbf{C}$ is abelian and that the simple $\mathbf{C}$-modules are finitely presented. All the above conditions on $\mathbf{C}$ and $\mathbf{D}=\bmod \mathbf{C}$ will be assumed throughout the paper.

Important cases where the above assumptions hold are $\mathbf{D}=\bmod \Lambda$, the category of finitely generated (left) modules over a left artin ring $\Lambda$, and the case where $\mathrm{D}$ is a dualizing $R$-variety for a commutative artin ring $R$ (see [4] for definition).

Let $\mathbf{D} / \mathbf{P}$ denote the category $\mathbf{D}$ modulo projectives (see [4]). We say that $\mathbf{D}=\bmod \mathbf{C}$ and $\mathbf{D}^{\prime}=\bmod \mathbf{C}^{\prime}$ are (projectively) stably equivalent if $\mathbf{D} / \boldsymbol{P}$ and $\mathbf{D}^{\prime} / \boldsymbol{P}$ are equivalent categories. For dualizing $R$-varieties this coincides with our previous definition of stable equivalence [4]. In [4] we discussed Loewy length for dualizing $R$-varieties $\mathrm{D}=\bmod \mathrm{C}$, denoted by $L L(\mathrm{D})$, coinciding with the ordinary Loewy length in the case of rings. We showed in [5] that if $\mathbf{D}=\bmod \mathbf{C}$ is a dualizing $R$-variety with $L L(\mathrm{D}) \leqslant 2$, there is a hereditary dualizing $R$-variety stably equivalent to $D$. In this paper we shall obtain this result in a completely different way. Our method here is interesting because we at the same time get a direct way of constructing a hereditary dualizing $R$-variety stably equivalent to a dualizing

Received by the editors August 16, 1974. $18 \mathrm{E} 10$.

AMS (MOS) subject classifications (1970). Primary 16A46, 16A62; Secondary 18A25, 
$R$-variety $\mathbf{D}=\bmod \mathbf{C}$ with $L L(\mathrm{D}) \leqslant 2$. Furthermore, our construction works in a more general context, namely with the assumptions on $\mathbf{D}=\bmod \mathbf{C}$ as above and $L L(D) \leqslant 2$. As for the definition of Loewy length it is easy to see that it can be defined in exactly the same way as for dualizing $R$-varieties in [4]. This more general case then has the advantage of applying to left artin rings with Loewy length at most 2 , rather than only to artin algebras (i.e. artin rings which are finitely generated as modules over their center). If $r$ denotes the radical of the artin ring $\Lambda$ then the hereditary ring we construct is $\Gamma=\left(\begin{array}{c}\Lambda / \mathrm{r} \\ \mathbf{r} \Lambda / \mathrm{r}\end{array}\right)$. This special case was treated in a preliminary way in [3].

In $\S 1$ we recall from [6] some background material on trivial extensions of abelian categories, and the definition and elementary properties of Loewy length from [4].

In $\S 2$ we define a new category $\mathbf{E}$ associated with $\mathbf{D}=\bmod \mathbf{C}$, which is going to be a hereditary abelian category with enough projectives stably equivalent to $\mathbf{D}$, and we define a natural functor $\alpha$ from $\mathbf{D}$ to $\mathbf{E}$. We then prove that $\alpha$ induces an equivalence between the categories modulo projectives for $\mathbf{D}$ and $\mathbf{E}$. We give a different description of $\alpha$ in the special case of a trivial extension $\mathbf{S} \ltimes F$ of a category $\mathbf{S}$ where each object is a finite direct sum of simple objects by a right exact functor $F: \mathbf{S} \rightarrow \mathbf{S}$.

In $\S 3$ we consider the natural ring map $n: \Gamma=\Lambda \times M \rightarrow \Delta=\left(\begin{array}{ll}\Lambda & 0 \\ M & \Lambda\end{array}\right)$, where $n(\lambda, m)=\left(\begin{array}{ll}\lambda & 0 \\ m & \lambda\end{array}\right)$. We assume that $\Lambda$ is a left artin ring and $M$ a $\Lambda$-bimodule, finitely generated as a left module. $n$ induces a natural functor $\beta: \bmod \Gamma \rightarrow$ $\bmod \Delta$, given by $\beta(X)={ }_{\Delta} \Delta_{\Gamma} \otimes_{\Gamma} X$, where the right action of $\Gamma$ on $\Delta$ is given via $n$. We shall see that although $\alpha$ and $\beta$ are different functors, they are isomorphic modulo projectives, so that also $\beta: \bmod \Gamma \rightarrow \bmod \Delta$ will induce an equivalence of the categories modulo projectives, when $\Lambda$ is semisimple (so that $L L(\Gamma) \leqslant 2$ ). We shall also consider the natural functor $\gamma: \bmod \Delta \rightarrow \bmod \Gamma$, which is given by considering a $\Delta$-module as a $\Gamma$-module via the map $n$. In particular we show that if $\Gamma$ is of finite representation type, then so is $\Delta$. We consider analogues of both $\beta$ and $\gamma$ in our more general category situation.

In $\$ 4$ we restrict ourselves to the case of dualizing $R$-varieties. We use our previous results [5] that if two hereditary dualizing $R$-varieties, both with no semisimple direct factors, are stably equivalent, then the hereditary dualizing $R$ varieties are equivalent. We shall show how to construct directly, on the basis of $\mathbf{D}=\bmod \mathbf{C}$, an hereditary dualizing $R$-variety $\mathbf{E}^{\prime}$ stably equivalent to $\mathbf{D}$ with no semisimple direct factors. We use this to formulate necessary and sufficient conditions for two dualizing $R$-varieties $\mathbf{D}=\bmod \mathbf{C}$ to be stably equivalent.

I would like to thank Professor Maurice Auslander for his helpful comments.

1. In this section we shall recall some background material on trivial extensions of abelian categories from [6], and on radical and Loewy length from [4]. 
Let $\mathbf{A}$ be an abelian category with enough projectives and $F: \mathbf{A} \rightarrow \mathbf{A}$ a right exact functor. We consider the trivial extension of $\mathbf{A}$ by $F$, denoted by $A \ltimes F$, which is then an abelian category. The objects are triples $(F A, A, f)$, where $f: F A \rightarrow A$ is a map in A such that $(F f) f=0$. We shall sometimes write

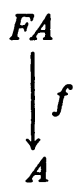

instead of $(F A, A, f)$. The projective objects are all of the form

$$
\begin{gathered}
F P+F^{2} P \\
\| \\
P \amalg F P
\end{gathered}
$$

for $P$ a projective object in $A$. The maps between two objects $(F A, A, f)$ and $(F B, B, g)$ are maps $h: A \rightarrow B$ such that

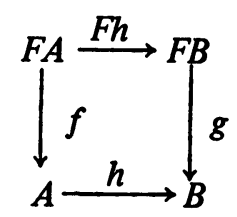

commutes.

As an important special case we have the case when $\mathbf{A}$ is the product of two abelian categories, $\mathbf{A}=\mathbf{B} \ltimes \mathbf{C}$, and $F: \mathbf{A} \rightarrow \mathbf{A}$ maps $\mathbf{B}$ to $\mathbf{C}$ and $\mathbf{C}$ to zero. We shall then usually denote the category $\mathbf{A} \ltimes F$ by $(B, C, F)$, and it will then be more convenient to denote the objects by $(B, C, f)$, where $f: F B \rightarrow C$ is a map in C. The projective objects are then direct sums of objects of the form $(P, F P$, id $)$ and $(0, Q, 0)$, where $P$ is projective in $\mathrm{B}$ and $Q$ projective in $\mathrm{C}$. The maps between $(B, C, f)$ and $\left(B^{\prime}, C^{\prime}, f^{\prime}\right)$ are pairs $\left(h_{1}, h_{2}\right)$, with $h_{1}: B \rightarrow B^{\prime}$ in B and $h_{2}: C \rightarrow C^{\prime}$ in $\mathbf{C}$, such that

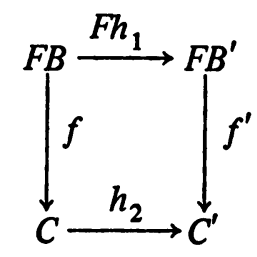

commutes.

We point out that an example of the above is given by $\mathbf{A}=\bmod \Lambda$, the category of finitely generated (left) modules over a left artin ring $\Lambda$, and $F=$ ${ }_{\Lambda} M_{\Lambda} \otimes-$, where $M$ is a two sided $\Lambda$-module which is finitely generated as a left $\Lambda$-module. Then $\bmod \Lambda \propto F$ is equivalent to the category $\bmod \Gamma$, where $\Gamma=$ $\Lambda \ltimes M$ is the trivial extension of $\Lambda$ by $M$, i.e. the ring whose elements are pairs 
$(\lambda, m)$ with $\lambda \in \Lambda$ and $m \in M$. Addition is componentwise, and multiplication is given by $(\lambda, m) \cdot\left(\lambda^{\prime}, m^{\prime}\right)=\left(\lambda \lambda^{\prime}, \lambda m^{\prime}+m \lambda^{\prime}\right)$.

An example of the special case $(\mathrm{B}, \mathrm{C}, F)$ is $(\bmod R, \bmod S, F)$, where $R$ and $S$ are left artin rings, $S_{R}$ is an $S-R$-bimodule which is a finitely generated $S$-module, and $F: \bmod R \rightarrow \bmod S$ is given by $F={ }_{s} M_{R} \otimes-$. If we write $\Gamma=\left(\begin{array}{cc}R & 0 \\ M & S\end{array}\right)$, where the ring elements are $\left(\begin{array}{cc}r & 0 \\ m & s\end{array}\right)$, with $r \in R, s \in S, m \in M$, addition is componentwise and

$$
\left(\begin{array}{ll}
r & 0 \\
m & s
\end{array}\right) \cdot\left(\begin{array}{ll}
r^{\prime} & 0 \\
m^{\prime} & s^{\prime}
\end{array}\right)=\left(\begin{array}{cc}
r^{\prime} & 0 \\
m r^{\prime}+s m^{\prime} & s s^{\prime}
\end{array}\right)
$$

then $(\bmod R, \bmod S, F)$ is equivalent to $\bmod \Gamma$.

We shall next recall some properties of radical and Loewy length from [4]. For a dualizing $R$-variety $\mathbf{D}=\bmod \mathbf{C}, \mathrm{r} M$ was defined to be the intersection of the maximal $\mathrm{C}$-submodules of $M$, for a finitely presented $\mathrm{C}$-module $M$. We showed various properties about $\mathrm{r} M$, similar to those known for rings. In particular, $\mathrm{r} M$ is also finitely presented, so that we can repeat the process in mod $\mathbf{C}$, to define $\mathrm{r}^{i} M$ for all $i \geqslant 1$. The smallest $i$ such that $\mathrm{r}^{i} M=(0)$ is called the Loewy length of $M$, denoted by $L L(M)$. The Loewy length of $\mathbf{D}=\bmod \mathbf{C}$ is the supremum of the Loewy length of all objects in $\mathbf{D}$, denoted by $L L(D)$. It is easy to see that all this can be done in our more general situation. The assumption that the simple C-modules are finitely presented is essential.

We shall be mainly concerned with the case $L L(D) \leqslant 2$. Then for each finitely presented C-module $M, \mathrm{r} M$ is finitely presented, and since $\mathrm{r}(\mathrm{r} M)=0, \mathrm{r} M$ is a direct sum of a finite number of simple objects in $\bmod C$. Since $M / \mathrm{r} M$ is also the direct sum of a finite number of simple objects, $M$ has finite length. We can now conclude that $\mathbf{D}=\bmod \mathbf{C}$ has projective covers. For since each object $(, C)$ has finite length, it is not hard to see that $\bmod \mathbf{C}^{\prime}$ is equivalent to $\bmod \Lambda$ for an artin ring $\Lambda$, where $\mathbf{C}^{\prime}$ is a finitely generated subvariety of $\mathbf{C}$ (i.e. $\mathbf{C}^{\prime}$ has only a finite number of indecomposable objects). Since each such mod $\mathbf{C}^{\prime}$ has projective covers, we can conclude by [2] that $\mathbf{D}=\bmod \mathbf{C}$ has projective covers.

2. We let the assumptions on $\mathbf{D}=\bmod \mathbf{C}$ be as before, and assume also that $L L(D) \leqslant 2$. In this section we shall show that $\mathrm{D}$ is projectively stably equivalent to some hereditary abelian category $\mathbf{E}$ with enough projectives, i.e. the categories $\mathrm{D} / P$ and $\mathrm{E} / P$ are equivalent.

We start by defining the category $\mathbf{E}$. Let $\mathbf{S}$ be the full additive subcategory of $\mathbf{D}$ whose indecomposable objects are the simple objects in $\mathbf{D}$. $\mathbf{S}$ is clearly abelian. We want to define a functor $F: \mathbf{S} \rightarrow \mathbf{S}$ as follows. For an object $S$ in $\mathrm{S}$, let $P_{S}$ denote its projective cover in $\mathrm{D}$, which we have seen exists. Define $F(S)=\mathrm{r} P_{S}$. If $f: S \rightarrow T$ in $S$, consider the diagram 


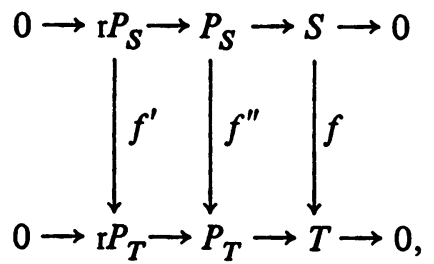

where $f^{\prime \prime}$ is a lifting of $f$, and $f^{\prime}$ its restriction to $\mathrm{r} P_{S}$. We then define $F(f)=$ $f^{\prime}: \mathrm{r} P_{S} \rightarrow \mathrm{r} P_{T}$. It is easily checked that $f^{\prime}$ is independent of the choice of $f^{\prime \prime}$.

If $0 \rightarrow S \rightarrow S \rightarrow S^{\prime \prime} \rightarrow 0$ is an exact sequence in $S, 0 \rightarrow F\left(S^{\prime}\right) \rightarrow$ $F(S) \rightarrow F\left(S^{\prime \prime}\right) \rightarrow 0$ is clearly exact, so that $F$ is an exact functor. (S, S, F) is then an abelian category, which obviously has enough projectives, and is hereditary [6].

In the special case $D=\bmod \Lambda$ for a left artin ring $\Lambda$, it is easily seen that we have $F(S)=\mathrm{r} P_{S} \cong \mathrm{r} \otimes S$. Hence $\bmod \Lambda$ is here equivalent to $\bmod \Gamma$, where $\Gamma=\left(\begin{array}{cc}\Lambda / \mathrm{r} & 0 \\ \mathrm{r} & \Lambda / \mathrm{r}\end{array}\right)$.

We next want to define a natural functor $\alpha: \mathbf{D} \rightarrow \mathbf{E}=(\mathbf{S}, \mathbf{S}, F)$. For an object $M$ in $\mathbf{D}$, define $\alpha(M)=(M / \mathrm{r} M, \mathrm{r} M, f)$, where $f: F(M / \mathrm{r} M) \rightarrow \mathrm{r} M$ is given as follows. Let $P_{M}$ be a projective cover of $M$. We know that $F(M / \mathrm{r} M)=\mathrm{r} P_{M}$, and we define $f: \mathrm{r} P_{M} \rightarrow \mathrm{r} M$ to be the epimorphism induced by the epimorphism $P_{M} \rightarrow M$. If $g: M \rightarrow N$ is a map in $\mathrm{D}$, then $\alpha(g)=\left(g_{1}, g_{2}\right)$, where $g_{1}: M / \mathrm{r} M \rightarrow$ $N / \mathrm{r} N$ and $g_{2}: \mathrm{r} M \rightarrow \mathrm{r} N$ are the natural maps induced by $g: M \rightarrow N$.

Let $\mathbf{E}^{\prime}$ denote the full subcategory of $\mathbf{E}$ whose objects are $(S, T, f)$, where $f: F S \rightarrow T$ is an epimorphism. We have seen that for $M$ in $\mathbf{D}, \alpha(M)$ lies in $\mathbf{E}^{\prime}$. We let $\alpha$ also denote the induced functor from $\mathbf{D}$ to $\mathbf{E}^{\prime}$. We have the following close connection between $\mathbf{D}$ and $\mathbf{E}^{\prime}$.

Proposition 2.3. $\alpha: \mathbf{D} \rightarrow \mathbf{E}^{\prime}$ is a representation equivalence, i.e. $\alpha$ is a full and dense functor such that if $\alpha(g)$ is an isomorphism then $g$ is an isomorphism.

Proof. Let $g: M \rightarrow N$ be a map in D. Then the diagram

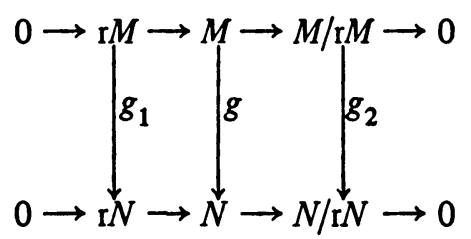

shows that if $F(g)=\left(g_{1}, g_{2}\right)$ is an isomorphism, i.e. both $g_{1}$ and $g_{2}$ are isomorphisms, then $g$ is an isomorphism.

If $\mathbf{C}$ is finitely generated, i.e. $\mathbf{D}=\bmod \mathbf{C}$ is equivalent to $\bmod \Lambda$ for some artin ring $\Lambda$, then dually to [1, Chapter 2], one can show that $\alpha$ is full and dense. We can use this special fact to show that $\alpha$ is full and dense also in the general case. We sketch below how to show that $\alpha$ is dense. The method for showing 
that $\alpha$ is full is similar. Let $(A, B, f)$ be an object in $\mathbf{E}^{\prime}$, i.e. $A$ and $B$ are semisimple objects in $\mathrm{D}$, and $f: \mathrm{r} P_{A} \rightarrow B$ is an epimorphism. Let $\left(, C_{2}\right) \rightarrow\left(, C_{1}\right)$ $\rightarrow\left(, C_{0}\right) \rightarrow A \rightarrow 0$ and $\left(, C_{1}^{\prime}\right) \rightarrow\left(, C_{0}^{\prime}\right) \rightarrow B \rightarrow 0$ be beginnings of minimal projective resolutions for $A$ and $B$ in $\mathbf{D}$.

Let $\mathbf{C}_{\mathbf{1}}$ be a full additive subcategory of $\mathbf{C}$, with only a finite number of indecomposable objects, containing the $C_{i}$ and $C_{i}^{\prime}$ above. Consider the natural right exact embedding $i: \bmod \mathrm{C}_{1} \rightarrow \bmod \mathrm{C}$, determined by $i(, C)=(, C)$ for all $C$ in $\mathbf{C}_{1}$. Denote by $j: \bmod \mathbf{C} \rightarrow \bmod \mathbf{C}_{1}$ the natural restriction functor, which is clearly exact. $j i: \bmod C_{1} \rightarrow \bmod C_{1}$ is isomorphic to the identity functor, and $i: \bmod \mathrm{C}_{1} \rightarrow \bmod \mathrm{C}$ induces an equivalence of categories between $\bmod \mathrm{C}_{1}$ and the full subcategory of mod $\mathbf{C}$, whose objects are the objects of mod $\mathbf{C}$ which have minimal projective presentations involving only projectives $(, C)$ with $C$ in $\mathbf{C}_{1}$ [2]. $A, B$ and $\mathrm{r}_{A}$ will then lie in this subcategory. Let $\mathbf{E}_{1}^{\prime}$ denote the category of triples $(X, Y, g)$, with $g: \mathrm{r} P_{X} \rightarrow Y$ an epimorphism, associated with the category $\bmod \mathrm{C}_{1}$ in the same way as before, i.e. $X$ and $Y$ are semisimple objects in $\bmod \mathbf{C}_{1}$, and $P_{X}$ denotes a projective cover of $X$ in $\bmod \mathbf{C}_{1}$. Denote the natural functor by $\alpha_{1}: \bmod \mathbf{C}_{1} \rightarrow \mathbf{E}_{1}$. We claim that $(j(A), j(B), j(f))$ lies in $\mathbf{E}_{1}$. We get induced an epimorphism $j\left(\mathrm{r} P_{A}\right) \rightarrow j(B)$. $j(A)$ and $j(B)$ are clearly semisimple objects in $\bmod \mathrm{C}_{1}$, so we need only check that $j\left(\mathrm{r} P_{A}\right) \cong \mathrm{r} P_{j(A)}$. This follows since it is easy to see that $P_{j(A)}$ is the projective cover of $j(A)$ in mod $\mathrm{C}_{1}$. As we already pointed out, since $\bmod \mathrm{C}_{1}$ is equivalent to $\bmod \Lambda$ for an artin ring $\Lambda$, there is an $M$ in mod $C_{1}$ such that $\alpha^{\prime}(M)=(j(A), j(B), j(f))$. We claim that $\alpha(i(M)) \cong(A, B, f)$. We have the exact sequence

$$
\underset{\mathbb{R}}{0 \rightarrow \mathrm{r} M \rightarrow M} \rightarrow \underset{\mathbb{R}}{M} \mathrm{r} M \rightarrow 0
$$

$j(B) \quad j(A)$

hence the right exact sequence $B \rightarrow i(M) \rightarrow A \rightarrow 0$. By applying $j$ to this sequence we get the above exact sequence back again. Since every nonzero subobject $B^{\prime}$ of $B$ is a direct summand of $B$, it is not hard to see that $j\left(B^{\prime}\right) \neq(0)$, and hence that the sequence $0 \rightarrow B \rightarrow i(M) \rightarrow A \rightarrow 0$ is actually exact. Since $A$ is semisimple, we have as in [5] for dualizing $R$-varieties, that $\operatorname{ri}(M) \subset B$. Since $j(A) \cong M / \mathrm{r} M$ in $\bmod \mathrm{C}_{1}, P_{j(A)}$ is also a projective cover for $M$. Hence $P_{A}$ is a projective cover for $i(M)$ in mod C. From this it is clear that $i(M) / \mathrm{ri}(M) \cong A$, hence also $\mathrm{ri}(M) \cong B$. Since $\mathrm{r} P_{A}$ and $B$ lie in the correct subcategory of $\bmod \mathbf{C}$, we have that $i j(f)=f: \mathrm{r} P_{A} \rightarrow B$. This completes the proof that $\alpha: \bmod \mathbf{C} \rightarrow \mathbf{E}^{\prime}$ is a dense functor.

We are now ready to formulate our main result.

THEOREM 2.4. In the above notation, $\alpha: \mathrm{D} \rightarrow \mathrm{E}=(\mathrm{S}, \mathrm{S}, F)$ induces an equivalence of categories between the categories $\mathrm{D} / \mathrm{P}$ ( $\mathrm{D}$ modulo projectives) and 
$\mathrm{E} / P$, i.e. $\mathrm{D}$ and $\mathbf{E}$ are projectively stably equivalent. Further $\mathbf{E}$ is hereditary.

Proof. Since by Proposition 2.3, $\alpha: \mathbf{D} \rightarrow \mathbf{E}^{\prime}$ is a representation equivalence, $\alpha(M)$ is indecomposable in $\mathbf{E}$ if and only if $M$ is indecomposable in $\mathbf{D}$. Let $M$ be an indecomposable object in D. Consider $\alpha(M)=(M / \mathrm{r} M, \mathrm{r} M, f)$. Here $f: \mathrm{r} P_{M} \rightarrow$ $\mathrm{r} M$ is an epimorphism which is an isomorphism if and only if $M$ is projective. By the description of projective objects in $\mathbf{E}=(\mathbf{S}, \mathbf{S}, F)$ which we recalled in $\S 1$, we can then conclude that $M$ is projective in $\mathbf{D}$ if and only if $\alpha(M)$ is projective in $\mathbf{E}$.

Let now $(A, B, f)$ be an indecomposable object in $\mathbf{E}$ which does not lie in $\mathbf{E}^{\prime}$, i.e. $f: \mathrm{r} P_{A} \rightarrow B$ is not an epimorphism. Since $B$ is semisimple, the monomorphism $\operatorname{Im} f \rightarrow B$ splits. Since $(A, B, f)$ is indecomposable, we can then conclude that $f$ is zero and $A=(0)$, so that $(A, B, f)=(0, B, 0)$, hence a projective object in $\mathbf{E}$, since $B$ is projective in $\mathbf{S}$. Hence $\alpha$ induces a full and dense functor, also denoted by $\alpha$, from the full subcategory of $\mathbf{D}$ whose objects are the objects of $\mathbf{D}$ with no projective summands, to the full subcategory of $\mathbf{E}$ whose objects are the objects in $\mathbf{E}$ with no projective summands. Since this $\alpha$ is full, we can write $\operatorname{Hom}_{\mathrm{E}}(\alpha(M), \alpha(N))=\operatorname{Hom}_{\mathrm{D}}(M, N) / S(M, N)$, where $S(M, N)$ is the subgroup of $\operatorname{Hom}_{\mathrm{D}}(M, N)$ whose elements are the maps $g: M \rightarrow N$ such that the induced maps $g^{\prime}: M / \mathrm{r} M \rightarrow N / \mathrm{r} N$ and $g^{\prime \prime}: \mathrm{r} M \rightarrow \mathrm{r} N$ are both zero, i.e. such that $g(M) \subset \mathrm{r} N$.

$\mathbf{E}$ is hereditary since gl. $\operatorname{dim} . \mathbf{S}=0[6]$, hence $\mathbf{E} / P$ is easily seen to be a full subcategory of $\mathbf{E}$. Hence the following lemma will finish the proof that $\alpha$ induces an equivalence of categories between $\mathrm{D} / P$ and $\mathrm{E} / P$.

LEMMA 2.5. Let $M$ and $N$ be objects in $\mathrm{D}$ with no projective summands. Then $S(M, N)=P(M, N)$, where $P(M, N)=\{f: M \rightarrow N$, where $f$ factors through a projective object in $\mathrm{D}\}$.

Proof. Assume first that $f \in P(M, N)$, i.e. we have a commutative diagram

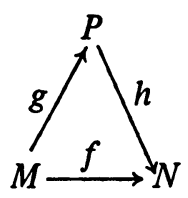

where $P$ is a projective object in D. Since $M$ has no projective summands, $g(M) \subseteq$ $\mathrm{r} P$. Hence $h g(M)=f(M) \subseteq \mathrm{r} N$, which implies that $f$ is in $S(M, N)$.

Assume conversely that $f \in S(M, N)$, i.e. $f$ factors as follows: $M \rightarrow M / \mathrm{r} M$ $\stackrel{f^{\prime}}{\longrightarrow} \mathrm{r} N \rightarrow N$. Let $g: P \rightarrow N$ be a projective cover for $N$. $g$ induces an epimorphism $g^{\prime}: \mathrm{r} P \rightarrow \mathrm{r} N$. Since $\mathrm{r} P$ is semisimple, there is an $h: M / \mathrm{r} M \rightarrow \mathrm{r} P$ which makes the diagram

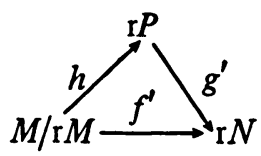


commute. It then follows that $f$ factors through $P$, so that $f$ is in $P(M, N)$. This finishes the proof of the lemma, and hence of the theorem.

COROLlary 2.7. Let $\Lambda$ be a left artin ring with $\mathrm{r}^{2}=0$. Then $\Lambda$ is (projectively) stably equivalent to the hereditary ring $\Gamma=\left(\begin{array}{cc}\Lambda / \mathrm{r} & 0 \\ \mathrm{r} \Lambda / \mathrm{r}\end{array}\right)$.

Proof. Let $\mathbf{D}=\bmod \Lambda$. Then the full additive subcategory of $\mathbf{D}$ whose indecomposable objects are the simple objects of $D$ is $\bmod \Lambda / r$. And we have seen that our functor $F: \bmod \Lambda / \mathrm{r} \rightarrow \bmod \Lambda / \mathrm{r}$ is given by $F(M)=\mathrm{r} \otimes_{\Lambda / \mathrm{r}} M$. The rest follows from our remarks in $\S 1$.

Corollary 2.8. Let $\mathbf{D}=\bmod \mathbf{C}$ where $L L(D) \leqslant 2$, and let $\mathbf{E}$ be as above. Then there is a one-one correspondence between the indecomposable nonprojective objects in $\mathbf{D}$ and $\mathbf{E}$. In particular, there is a finite number of indecomposable nonprojective objects in $\mathbf{D}$ if and only if there is a finite number of indecomposable nonprojective objects in $\mathbf{E}$.

We end this section by discussing an important class of our $\mathbf{D}=\bmod \mathbf{C}$ with $L L(D) \leqslant 2$. Let $\mathrm{S}$ be an abelian category where each object is a finite sum of simple objects, and $F: \mathbf{S} \rightarrow \mathbf{S}$ a (right) exact functor. Let $\mathbf{D}=\mathbf{S} \ltimes F=$ mod $\mathbf{C}$ (assuming that $\mathbf{D}$ is a category of the type we consider in this paper). For an object $(F A, A, f)$ in $\mathbf{S} \ltimes F$, we have the exact sequence

$$
0 \rightarrow(F(\operatorname{Im} f), \operatorname{Im} f, 0) \rightarrow(F A, A, f) \rightarrow(F(\text { Coker } f), \text { Coker } f, 0) \rightarrow 0
$$

[6]. For $0 \rightarrow \operatorname{Im} f \rightarrow A \rightarrow$ Coker $f \rightarrow 0$ is exact in $S$, so the only thing left to show is that the induced maps $F(\operatorname{Im} f) \rightarrow \operatorname{Im} f$ and $F($ Coker $f) \rightarrow$ Coker $f$ are both zero. The first follows from

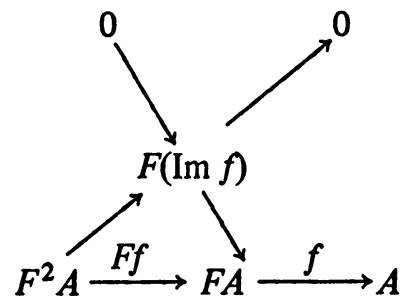

where $f(F f)$ is zero. Further $F A \stackrel{f}{\rightarrow} A \rightarrow$ Coker $f$ is clearly zero, and the map $F A \stackrel{f}{\rightarrow} A$ factors through $F($ Coker $f)$ since $f(F f)$ is zero.

We consider the natural embedding of $\mathrm{S}$ into $\mathrm{S} \ltimes F$ given by $S \rightarrow(F S, S, 0)$ for $S$ in $S$ an identification. If $M=(F A, A, f)$ is an indecomposable nonsimple object, it is not hard to see that $\mathrm{r} M=(F(\operatorname{Im} f), \operatorname{Im} f, 0)$. We now want to com- 
pute $\mathrm{r} \boldsymbol{P}_{M}$. It is easy to see that

$$
P_{M}=\begin{gathered}
F(\operatorname{Coker} f) \amalg F^{2}(\operatorname{Coker} f) \\
\text { Coker } f \amalg F(\operatorname{Coker} f)
\end{gathered}
$$

[6], hence $\mathrm{r} P_{M}=\left(F^{2}(\operatorname{Coker} f), F(\operatorname{Coker} f), 0\right)$. It will then follow that $\alpha(M)=$ (Coker $\left.f, \operatorname{Im} f, f^{\prime}\right)$, where $f^{\prime}: F($ Coker $f) \rightarrow \operatorname{Im} f$ is induced by $f: F A \rightarrow A$. We state this as

Proposition 2.9. Let $\mathbf{S}$ be an additive category where each object is a finite direct sum of simple objects, and let $F: \mathbf{S} \rightarrow \mathbf{S}$ be a right exact functor. Then $\alpha: \mathbf{D}=\mathbf{S} \times F \rightarrow(\mathbf{S}, \mathbf{S}, F)=\mathbf{E}$ given by $\alpha(A, F A, f)=\left(\operatorname{Coker} f, \operatorname{Im} f, f^{\prime}\right)$, with $f^{\prime}: F(\operatorname{Coker} f) \rightarrow \operatorname{Im} f$ the natural map induced by $f: F A \rightarrow A$, induces an equivalence of categories between $\mathrm{D} / P$ and $\mathrm{E} / P$.

We remark that for the case of a trivial extension $\mathbf{A} \ltimes F$, where $\mathbf{A}$ is not semisimple, the above functor $\alpha$ generalizes to a functor $\alpha^{\prime}: \mathbf{A} \propto F \rightarrow(\mathbf{A}, \mathbf{A}, F)$ given by $\alpha^{\prime}(A, F A, f)=\left(\right.$ Coker $\left.f, \operatorname{Im} f, f^{\prime}\right)$.

3. Let $\Lambda$ be a left artin ring and $M$ a twosided $\Lambda$-module which is finitely generated as a left $\Lambda$-module. As a special case of our considerations in the previous section we have a natural functor $\alpha^{\prime}: \bmod \Gamma \rightarrow \bmod \Lambda$, where $\Gamma=\Lambda \ltimes M$ and $\Delta=\left(\begin{array}{l}\Lambda \\ M\end{array}\right)$. If $\Lambda$ is semisimple, we showed that $\alpha$ induced an equivalence of the categories mod $\Gamma$ and $\bmod \Lambda$ modulo projectives.

Associated with the natural ring map $n: \Gamma \rightarrow \Delta$, given by $n(\lambda, m)=\left(\begin{array}{ll}\lambda & 0 \\ m & \lambda\end{array}\right)$ are the functors $\beta: \bmod \Gamma \rightarrow \bmod \Delta$, given by $\beta(M)={ }_{\Delta} \Delta_{\Gamma} \otimes M$ for $M$ in $\bmod \Gamma$, where $\Delta$ is considered a right $\Gamma$-module via the map $n$, and $\gamma: \bmod \Delta \rightarrow \bmod \Gamma$, where for $N$ in $\bmod \Delta, \gamma(N)$ is a $\Gamma$-module via the map $n$.

We shall compare the functors $\alpha^{\prime}$ and $\beta$, which turn out to be different in general, but for the case of $\Lambda$ being semisimple, the functors induced by $\alpha$ and $\beta$ from $\bmod \Gamma / P$ to $\bmod \Delta / P$ are isomorphic. So the stable equivalence between $\bmod \Gamma$ and $\bmod \Delta$ is in this case induced by a natural tensor product.

We have seen that when $\Gamma$ and $\Delta$ are stably equivalent then $\Gamma$ is of finite representation type if and only if $\Delta$ is. We shall use the functor $\boldsymbol{\gamma}$ to show that even if $\Lambda$ is not semisimple, we can still conclude that if $\Delta$ is of finite representation type, then $\Gamma$ is.

We shall, more generally, consider an abelian category $\mathbf{A}$ with enough projectives and the categories $\mathbf{A} \ltimes F$ and $(\mathbf{A}, \mathbf{A}, F)$ (subject to our standard assumptions for this paper), where $F: \mathbf{A} \rightarrow \mathbf{A}$ is a right exact functor. We want to define functors $\beta: \mathbf{A} \ltimes F \rightarrow(\mathbf{A}, \mathbf{A}, F)$ and $\gamma:(\mathbf{A}, \mathbf{A}, F) \rightarrow \mathbf{A} \ltimes F$ such that they specialize to the above $\beta$ and $\gamma$ for rings. 
Proposition 3.1. We have a right exact functor $\beta: \mathbf{A} \ltimes F \rightarrow(\mathbf{A}, \mathbf{A}, F)$ given by $\beta(F A, A, f)=\left(\operatorname{Coker} f, A, f^{\prime}\right)$, where $f^{\prime}: F($ Coker $f) \rightarrow A$ is naturally induced by $f: F A \rightarrow A$. And if $g:(F A, A, f) \rightarrow(F B, B, h)$, then $\beta(g)=\left(g^{\prime}, g\right)$, where $g^{\prime}:$ Coker $f \rightarrow$ Coker $h$ is induced by $g: A \rightarrow B$.

Proof. We have already seen in $\S 2$ that $f: F A \rightarrow A$ induces a map $f^{\prime}: F(\operatorname{Coker} f) \rightarrow A$. Consider then $g:(F A, A, f) \rightarrow(F B, B, h)$, i.e. we have a commutative diagram

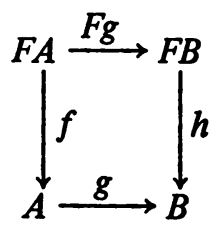

It is then clear that

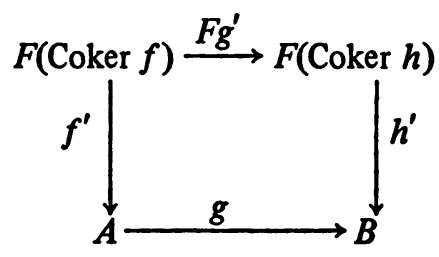

commutes, so that $\left(g, g^{\prime}\right)$ is indeed a map from (Coker $\left.f, A, f^{\prime}\right)$ to (Coker $h, B, h^{\prime}$ ). To show that $\beta$ is right exact, let $(F A, A, f) \rightarrow(F B, B, h) \rightarrow(F C, C, i) \rightarrow 0$ be exact in $\mathbf{A} \times F$. Then $A \rightarrow B \rightarrow C \rightarrow 0$ is exact in $A$, hence also $F A \rightarrow$ $F B \rightarrow F C \rightarrow 0$ since $F$ is a right exact functor. Then we get induced a right exact sequence Coker $f \rightarrow$ Coker $h \rightarrow$ Coker $i \rightarrow 0$, and we conclude that

$$
\left(\text { Coker } f, A, f^{\prime}\right) \rightarrow\left(\text { Coker } h, B, h^{\prime}\right) \rightarrow\left(\text { Coker } i, C, i^{\prime}\right) \rightarrow 0
$$

is exact in (A, A, F), and our proof is complete.

It is not hard to see that the functor $\beta$ in Proposition 3.1 generalizes the above mentioned $\beta$ for artin rings: Our notation of $\Lambda \times M$ as a $\Lambda \propto M$-module is

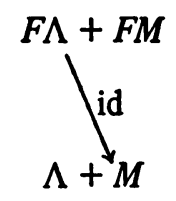

and of $\Delta=\left(\begin{array}{cc}\Lambda & 0 \\ M & \Lambda\end{array}\right)$ às a $\Delta$-module,<smiles>[Y10]CC</smiles>

Hence $\beta(\Gamma)=\Delta$. Since $\beta$ is right exact and commutes with sums, we know that 
$\beta$ is given by $\beta(X)={ }_{\Delta} \Delta_{\Gamma} \otimes X$, where the right structure of $\Delta$ as a $\Gamma$-module is given via the map $n$. For if $h: \Gamma \rightarrow \Gamma$ is given by right multiplication by $(\lambda, m)$, it is not hard to see that $\beta(f): \Lambda \rightarrow \Lambda$ is given by right multiplication by $\left(\begin{array}{ll}\lambda & 0 \\ m & \lambda\end{array}\right)$.

If $\mathbf{A}$ is semisimple, we have

$$
\begin{aligned}
\beta(F A, A, f) & =\left(\operatorname{Coker} f, A, f^{\prime}\right)=\left(\operatorname{Coker} f, \operatorname{Im} f, f^{\prime}\right) \amalg(0, \operatorname{Coker} f, 0) \\
& =\alpha(F A, A, f) \amalg(0, \operatorname{Coker} f, 0) .
\end{aligned}
$$

Since $(0$, Coker $f, 0)$ is a projective object in $(\mathbf{A}, \mathbf{A}, F)$, the functors induced by $\alpha$ and $\beta$ on $\bmod \Gamma / P$ are isomorphic. We have then proved

Proposition 3.2. Let $\mathbf{A} \times F,(\mathbf{A}, \mathbf{A}, F)$ and the functor $\beta$ be as before. If $\mathbf{A}$ is semisimple, then $\beta$ induces an equivalence of categories between the categories $\mathbf{A} \ltimes F$ and $(\mathbf{A}, \mathbf{A}, F)$ modulo projectives.

One can also check that $\gamma:(\mathbf{A}, \mathbf{A}, F) \rightarrow(\mathbf{A}, F)$ given by

$$
\gamma(A, B, f)=\sum_{A \amalg B}^{F A \amalg F B}
$$

and $\gamma\left(g_{1}, g_{2}\right)=g_{1} \amalg g_{2}$ when $\left(g_{1}, g_{2}\right):(A, B, f) \rightarrow\left(A^{\prime}, B^{\prime}, f^{\prime}\right)$ is a map in $(\mathrm{A}, \mathbf{A}, F)$ specializes to the above $\boldsymbol{\gamma}$ for the case of artin rings. The composite functor $\beta \gamma:(\mathbf{A}, \mathbf{A}, F) \rightarrow(\mathbf{A}, F)$ is then given by

$$
\begin{aligned}
\beta \gamma(A, B, f) & =\beta\left(\begin{array}{l}
F A \amalg F B \\
A \amalg B
\end{array}\right) \\
& =(A, B, f) \amalg(0, A, 0) \amalg(\text { Coker } f, 0,0) .
\end{aligned}
$$

Hence every object in $(\mathbf{A}, \mathbf{A}, F)$ is a summand of an object $\beta(N)$ with $N$ in $\mathbf{A} \ltimes F$. Hence if $\mathbf{A} \ltimes F$ has only a finite number of indecomposable objects, then so does $(A, A, F)$. As a special case we have

\section{Corollary 3.3. Let $\Lambda$ be a left artin ring, $M$ a two sided $\Lambda$-module,} finitely generated as a left $\Lambda$-module. If $\Gamma=\Lambda \propto M$ is of finite representation type, then so is $\Delta=\left(\begin{array}{cc}\Lambda & 0 \\ M & \Lambda\end{array}\right)$.

4. In this section we shall assume that $\mathbf{D}=\bmod \mathbf{C}$ is a dualizing $R$-variety, where $R$ is a commutative artin ring [4]. We shall also assume $L L(D) \leqslant 2$. It is then not hard to see that $\mathbf{E}=(\mathbf{S}, \mathbf{S}, F)$, in our previous notation, is also a dualizing $R$-variety. The idea for the proof of this is to use that for each indecomposable projective object $P$ in $\mathbf{E}$ there is only a finite number of nonisomorphic 
indecomposable objects in $\mathbf{E}$ with a nonzero map to $P$.

From [5] we know that up to equivalence of categories there is a unique hereditary dualizing $R$-variety with no semisimple direct factor, stably equivalent to a dualizing $R$-variety with Loewy length at most two. We want to show how to construct this hereditary dualizing $R$-variety from $\mathrm{D}=\bmod \mathrm{C}$ with $L L(\mathrm{D}) \leqslant 2$. We shall apply this to give a criterion for when two dualizing $R$-varieties with Loewy length at most two are stably equivalent.

Let $\mathrm{D}=\bmod \mathrm{C}$ be a dualizing $R$-variety of Loewy length at most two, and $S$ the full additive subcategory where the indecomposable objects are the simple objects in $\mathbf{D}$. Let $\mathbf{S}_{\mathbf{1}}$ denote the full additive subcategory of $\mathbf{S}$ whose indecomposable objects are the simple nonprojective objects in $\mathbf{D}$. Let $\mathbf{S}_{2}$ denote the full additive subcategory of $\mathbf{S}$ whose indecomposable objects are the simple noninjective objects in $\mathbf{D}$. Then $\mathbf{S}=\mathbf{S}_{1} \times \mathbf{S}_{1}^{\prime}=\mathbf{S}_{2} \times \mathbf{S}_{2}^{\prime}$, where the indecomposable objects in $\mathbf{S}_{1}^{\prime}$ are the projective simple objects in $\mathbf{D}$, and the indecomposable objects in $\mathbf{S}_{2}^{\prime}$ the injective simple objects in $\mathbf{D}$. If $S$ is semisimple and projective, then clearly $F(S)=0$, and no simple injective object in D can be a summand of any object of the type $F(S)$. Hence $\mathbf{E}=(\mathbf{S}, \mathbf{S}, F)$ decomposes as $\mathbf{E}=\left(\mathbf{S}_{1}, \mathbf{S}_{2}, F\right)$ $\times \mathbf{S}_{1}^{\prime} \times \mathbf{S}_{2}^{\prime}$. It is easy to see that $\left(\mathbf{S}_{1}, \mathbf{S}_{2}, F\right)$ can have no semisimple direct factor. We summarize this discussion in

THEOREM 4.1. Let $\mathrm{D}$ be a dualizing $R$-variety with $L L(\mathrm{D}) \leqslant 2$. Then $\mathrm{D}$ is stably equivalent to the hereditary dualizing $R$-variety $\left(\mathbf{S}_{1}, \mathbf{S}_{2}, F\right)$, where $\mathbf{S}_{1}$ is the full additive subcategory of $\mathbf{D}$ generated by the simple nonprojective objects, and $\mathbf{S}_{2}$ generated by the simple noninjective objects. Further, $\left(\mathbf{S}_{1}, \mathbf{S}_{2}, F\right)$ has no semisimple direct factors.

We shall now use Theorem 4.1 to describe directly when two dualizing $R$ varieties $\mathbf{D}$ and $\mathbf{D}^{\prime}$ with Loewy length at most two are stably equivalent. By the remarks from the introduction of this section, $\mathbf{D}$ and $\mathbf{D}^{\prime}$ are stably equivalent if and only if the dualizing $R$-varieties $\left(\mathbf{S}_{1}, \mathbf{S}_{2}, F\right)$ and $\left(\mathbf{S}_{1}^{\prime}, \mathbf{S}_{2}^{\prime}, F^{\prime}\right)$ as described in Theorem 4.1 are equivalent. This can be seen to be the case if and only if there are equivalences of categories $G_{1}: \mathrm{S}_{1} \rightarrow \mathrm{S}_{1}^{\prime}$ and $G_{2}: \mathrm{S}_{2} \rightarrow \mathrm{S}_{2}^{\prime}$, between the nonprojective objects and the noninjective objects respectively, such that we have natural isomorphisms $G_{2}(F(S)) \cong F^{\prime}\left(G_{1}(S)\right)$ for $S$ in $S_{1}$.

\section{REFERENCES}

1. M. Auslander, Representation dimension of artin algebras, Queen Mary College Notes, 1971.

2. - Representation theory of artin algebras. I, Comm. Algebra 1 (1974), 177268.

3. M. Auslander and I. Reiten, Stable equivalence of artin algebras, Proc. Conf. on Orders, Group Rings and Related Topics, 1973, pp. 8-70. 
4. M. Auslander and I. Reiten, Stable equivalence of dualizing $R$-varieties, Advances in Math. 12 (1974), 306-366.

5. - Stable equivalence of dualizing $\boldsymbol{R}$-varieties. III: Dualizing $\boldsymbol{R}$-varieties stably equivalent to hereditary $R$-varieties, Advances in Math. 17 (1975).

6. R. Fossum, P. Griffith and I. Reiten, Homological algebra of trivial extensions of abelian categories with applications to ring theory, Lecture Notes in Math., vol. 456, SpringerVerlag, Berlin and New York, 1975.

DEPARTMENT OF MATHEMATICS, UNIVERSITY OF TRONDHEIM, NLHT, 7000 TRONDHEIM, NORWAY 